\title{
Quality of Life as an Indicator for Care Delivery in Clinical Oncology Using FHIR
}

\author{
Chantal N.L. BEUTTER ${ }^{\mathrm{a}, \mathrm{b}, 1}$, Jan ROSS ${ }^{\mathrm{b}}$, Patrick WERNER ${ }^{\mathrm{b}}$, \\ Dilyana VLADIMIROVA ${ }^{\mathrm{c}}$, Uwe M. MARTENS ${ }^{\mathrm{b}, \mathrm{c}}$ and Christian FEGELER ${ }^{\mathrm{a}, \mathrm{b}}$

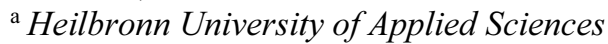 \\ ${ }^{\mathrm{b}}$ MOLIT Institute gGmbH \\ c SLK Clinics Heilbronn
}

\begin{abstract}
INTRODUCTION Health-related quality of life (HR-QoL) as a parameter for patient well-being is becoming increasingly important.[1] Nevertheless, it is mainly used as an endpoint in studies rather than as an indicator for adjustments in therapy. In this paper we will present an approach to gradually integrate quality of life $(\mathrm{QoL})$ as a control element into the care delivery of oncology. CONCEPT Acceptance, usability, interoperability and data protection were identified and integrated as key indicators for the development. As an initial approach, a questionnaire tool was developed to provide patients a simplified answering of questionnaires and physicians a clearer presentation of the results. IMPLEMENTATION As communication standard HL7 FHIR was used and known security concepts like OpenID Concept were integrated. In a usability study, first results were achieved by asking patients in the waiting room to answer a questionnaire, which will be discussed with the physician in the appointment. This study was conducted in 2019 at the SLK Clinics Heilbronn and achieved 86\% participation of all respondents with an average age of 67 years. DISCUSSION Although the evaluation study could prove positive results in usability and acceptance, it is necessary to aim for longitudinal surveys in order to include QoL as a control element in the therapy. However, a longitudinal survey through questionnaires leads to decreasing compliance and increasing response bias. [2] For this reason, the concept needs to be expanded. With sensors a continuous monitoring can be carried out and the data can be mapped to the individual, interpreted by machine learning. CONCLUSION Questionnaires are a concept that has been successfully applied in studies for years. However, since care delivery poses different challenges, the integration of new concepts is inevitable. The authors are currently working on an extension of the use of questionnaires with patient generated data through sensors.
\end{abstract}

Keywords. Quality of life, FHIR, monitoring, oncology, patient reported outcome, patient empowerment, patient generated data

\section{Introduction}

\subsection{Background}

According to the World Health Organization (WHO), quality of life (QoL) is the 'individual's perception of their position in life, in the context of the culture and value

${ }^{1}$ Corresponding Author, Chantal Beutter, MOLIT Institute gGmbH, Im Zukunftspark 10, 74076 Heilbronn, Germany; E-mail: Chantal.beutter@molit.eu. 
system in which they live and in relation to their goals, expectations, standards and concerns'. [3] In the context of treating patients, health-related quality of life (HR-QoL) becomes important. This includes a multidimensional concept that is related to the subjective perception of the patient about the effect of their disease and its treatment on the physical, psychological and social level of daily life. [4]

Health-related quality of life is becoming increasingly important in medicine, especially in oncology, where the disease itself, as well as the treatment leads to a strong reduction in QoL. Many studies have shown a positive influence of HR-QoL, not only on well-being, but also on survival time and a reduction in hospitalizations. [5 - 7] The aim of QoL research in oncology is to describe the current state of mind of a patient and, depending on this, to derive treatment strategies or therapy alternatives. [1] But up to now, HR-QoL has mainly been used as an outcome parameter in studies. Nevertheless, the final report of the further development of the Federal Ministry of Health (BMG) in Germany's eHealth strategy also shows that a disease is no longer defined and influenced exclusively as a purely physical illness, but also in terms of the patient's subjective wellbeing. [8]

Since QoL reflects the subjective perception of a person, patient empowerment, patient reported outcome (PRO) and also in some way patient generated data are of central importance in the use case of integrating HR-QoL into the treatment of the patient. The inclusion of mobile devices claims new, flexible communication standards, such as HL7 FHIR to enable that change of paradigm in healthcare. The MOLIT Institute has set itself the task of developing a tool to facilitate the integration of QoL into regular medical services in oncology. This is intended to improve the individual physician-patient relationship and to integrate $\mathrm{HR}-\mathrm{QoL}$ as an active control component in the therapy.

\subsection{Requirements}

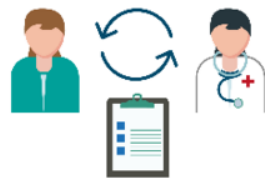

HR-QoL as a control parameter in therapy

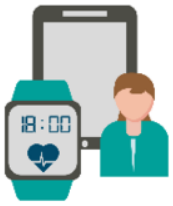

Patient-Empowerment through wearables

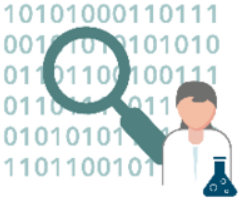

Secondary use of data for research

Figure 1: Schematic overview of the three identified requirements. As the main purpose HR-QoL needs to be integrated as a control parameter in the therapy. Next step is using patient generated data to continuously survey QoL. As an additional benefit data may be reused for data research.

Including HR-QoL into care delivery we have identified three requirements through literature or interviews with stakeholder. First of all, a change in the primary use towards using HR-QoL as a control parameter in the therapy of a patient, rather than as a rigid endpoint in a study needs to be done. QoL should influence the treatment context by giving the physician the opportunity to actively include a change in personal well-being in medical decisions.

As the next step, this use case should be extended with collected data from sensors of for example wearables of the patient. As mentioned above, FHIR gives the opportunity to include such devices and to transmit data in a structured and semantic interoperable 
way. This data should be processed and interpreted in the context of measuring HR-QoL and compared with a standard QoL measurement tool. From this, the next step is to generate knowledge about the validity of an alternative survey of HR-QoL using sensory analysis.

Last but not least, the collected data gives the opportunity to be reused for research, since it is structurally and semantically interoperable due to a uniformly used data structure.

In summary, the authors want to achieve a change in care delivery for the active inclusion of HR-QoL in therapy decisions in a concrete therapeutically situation, which brings further advantages by having electronically structured data.

\section{State of the art}

\subsection{Quality of Life}

In Germany, there are only few QoL measurement tools specific to cancer apart from the QLQ-C30 specifications. Internationally, there are multiple measurement methods, such as the Karnofsky- or Spitzer Index, or other questionnaires such as EQ-5D, SF-36 or SIRO, but it is noticeable that there is neither a national nor an international gold standard for measuring QoL.

The measurement of QoL can be divided into four different axes:

1. dimensionality

2. disease reference

3. source of information (self-assessment or external assessment)

4. behavioral reference

In most studies, QoL is assessed in a multidimensional, disease-related and selfassessment manner. However, the behavioral reference is usually deferred, which facilitates a response or fatigue bias in the collected data. [2]

Moreover, QoL is increasingly included as a primary endpoint, and it also plays an increasingly important role in pivotal studies. [1, 5-7] Nevertheless, QoL is rarely included as an endpoint or control component in regular medical services in clinics at this moment. So far, there is no data on a longitudinal collection and interpretation of HR-QoL data beyond studies.

\subsection{Interoperability in healthcare}

Health Level 7 (HL7) is a non-profit organization that has the mission to promote interoperability, i.e. data exchange, in the health care system. The communication standards developed by HL7 are internationally established and applied. [9] The communication standard HL7 v2 is considered the most implemented standard in health care worldwide. [10] FHIR as the newest standard of HL7 combines the advantages of the versions v2 and v3, as well as of CDA. With the support of mobile architectures for location-independent networking, increased transparency through open interfaces and the structuring of information for simplified further processing, the standard is becoming increasingly popular. $[10,11]$

FHIR is licensed as public domain to ensure free usage and easy access to the standard defining documents. The German Federal Association of Statutory Health 
Insurance Physicians (KBV) [11] opted for FHIR to implement the information objects of the federal electronic health record.

\section{Concept and methodology}

The first aim was to develop a tool that offers enough flexibility to connect the patient with the physician in an optimal way and to survey HR-QoL in a structured way. Thus, data can be optimally prepared and displayed for further analysis.

The first step was a literature review to analyze the current state of science and technology and to identify possible concepts for reuse. Common databases, such as PubMed or Google scholar were used with search terms like "monitoring quality of life", "quality of life oncology", "measurement quality of life" or similar.

Since QoL has so far been mainly determined by questionnaires, a concept for a questionnaire tool was developed. The tool should display questionnaires digitally and report the evaluation in a system especially designed for physicians. The initial use case was discussed and developed in cooperation with patients, as well as physicians. A usercentered design was chosen for a process that can be easily adapted by the stakeholders.

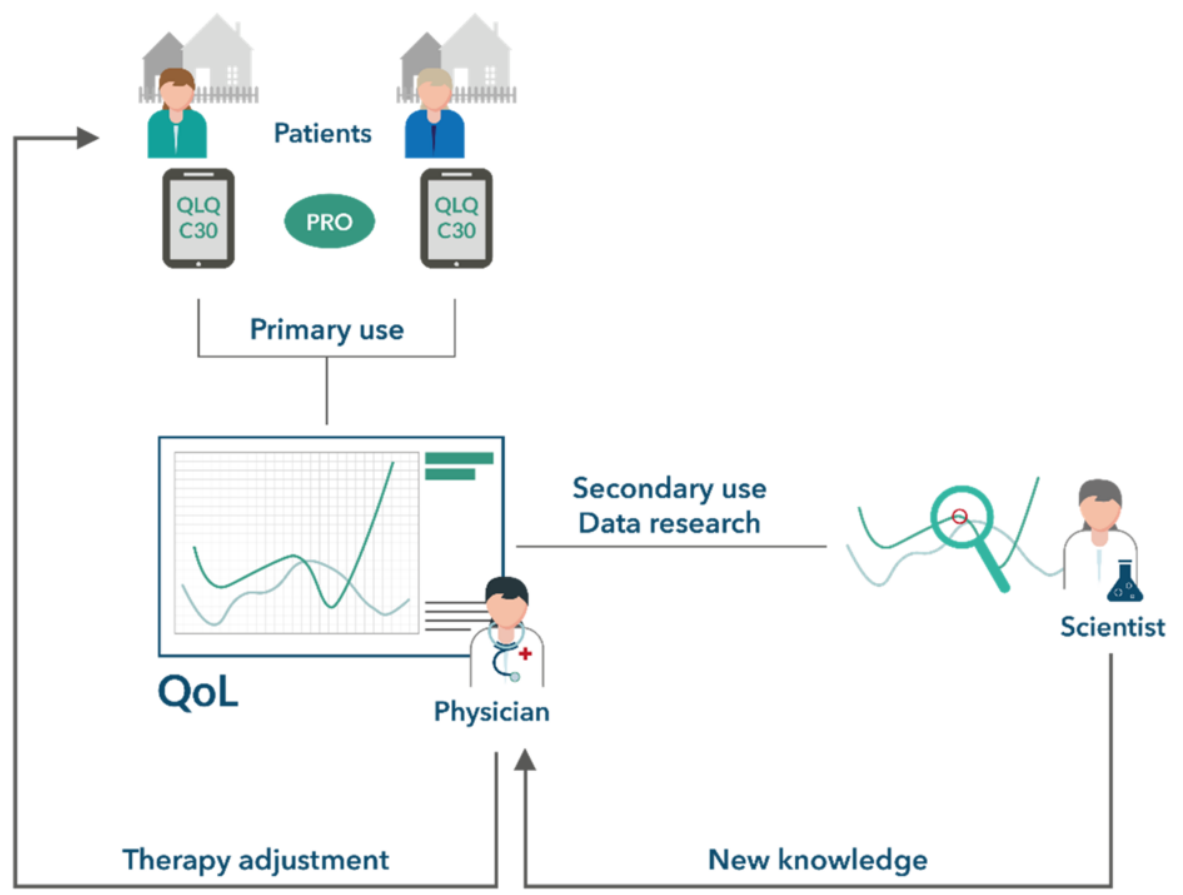

Figure 2: Workflow of the concept of the questionnaire tool. Patients may fill out questionnaires on mobile devices platform- and location independently so that the physician has the opportunity to adjust the therapy based on the given information. As a secondary use the structured data may be used for data research.

Initially patients may fill out the integrated questionnaire in the waiting room so that the data is forwarded to the physician's system via a hospital-internal server after completion. The physician therefore may view the data before the consultation in order to optimally respond to the patient's state of health. 
Patients should be able to fill out questionnaires on a mobile device, initially in the hospital, later from home, in order to integrate a continuous survey of HR-QoL into the treatment. Great importance was attached to interoperability with existing systems, a comprehensive security concept and good usability for better acceptance.

In the future we would like to provide a permanent monitoring of the psychological wellbeing of a patient. In accordance to the PDCA-cycle we would like to measure the influence of a patient's therapy on their HR-QoL to give physicians the opportunity to react on significant changes.

\section{Implementation}

In order to provide the flexibility and interoperability mentioned previously, the questionnaire tool was implemented in vue.js using HL7 FHIR as the communication standard. [12,13] This means that all questionnaires defined in this standard can be displayed platform- and hardware independently. Furthermore,FHIR simplifies the secondary use of data as it allows a semantic coding of questions.

In order to guarantee the security of the data, it is stored on an FHIR server implemented in the protected network of the respective hospital. Since patients fill out the questionnaire initially in the waiting room, none of the collected data leaves the hospital network at any time. Already established security concepts, such as OpenID Connect (OIDC) and OAuth2 for authentication, have been used.

By separating the content on the physician's system for evaluation and answering the questionnaires on an additional system on a mobile device, a minimum of sensitive data is stored on the device used. Running the system, only a list of the patients who have been assigned a questionnaire by the physician to answer, and this questionnaire itself is stored on the device. The answers are not saved there, but send directly on the clinical server. To display the questionnaires on the tablet, authentication using OIDC takes place when the program is initially started. The integrated patient list is protected with an additional code. This code is also activated if the tablet has been inactive for a certain period of time.

Patient data from the primary system can be integrated into the physician's system for a simplified process flow. The physician can search for patients specifically to assign new questionnaires or view answers already given. To enable a high usability, all interfaces and processes were designed in a collaboration with a graphic designer. The tool also gives the possibility to integrate a logic that reacts to certain conditions with defined responses.

\subsection{Preliminary results}

In a usability study at the SLK Clinics in Heilbronn the initial use case was evaluated. The aim was to estimate if the user centered approach lead to high acceptance of the implementation. 92 patients of the oncological outpatient clinic of the SLK Clinics participated over 3 weeks in March 2019. 108 patients were asked to voluntarily fill out a questionnaire (in this case the EQ 5D) on a tablet in the waiting room while waiting for their appointment. Before participating they received an information sheet about the aims and data protection of the system. The answers then were discussed with the physician at the consultation. After that the participants were asked to evaluate the tool. $86 \%$ of all respondents agreed to participate and evaluate the tool. They were given a self-designed 
questionnaire, combining parts of standardized measuring tools, like the elements mentioned in the ISO9241-11 or the Usability Experience Questionnaire (UEQ), enlarged with some additional questions scaled on the use case. The average age was 67 years, with the oldest participant being 88 years old. $86 \%$ could imagine the integration of the tool into their everyday life, $37 \%$ of which stated that they had never or rarely worked with mobile devices. $82 \%$ saw an additional value in using the tool and $94 \%$ rated the process flow as well designed and time-saving. Suggestions were, for example, the expansion of the system for answering at home or the connection of private mobile devices.

Due to the positive patient response, the questionnaire tool EQU was integrated into the regular medical care of oncology within the SLK Clinics Heilbronn.

\section{Lessons learned (Discussion)}

The results of the pilot study in the SLK Clinics Heilbronn show that focusing on the key indicators mentioned above leads to a good acceptance, even for technically inexperienced patients. The feedback had also shown that good patient education about data protection and data security of the system increases the willingness to use it. Many patients only agreed their participation after an understanding of the importance of the collection of data and its security. In the overall picture it could be determined that there is a willingness to integrate digital concepts. Nevertheless, German hospital systems are currently still in the early stages of adopting FHIR. As already mentioned, v2 is still the most used communication standard in Germany. Therefore, the proposed questionnaire solution requires a FHIR - v2 adapter or has to be integrated through a communication server. Since v2 does not support questionnaires new solutions are required. Due to the flexibility of the standard, it has been decided for FHIR.

If we apply the axes mentioned at the beginning to our concept, however, more limitations become clear:

1. dimensionality

The dimensionality for recording QoL depends on the questionnaires used. The system does not impose any restrictions in this respect.

2. disease reference

The disease reference also depends on the questionnaire. The system thus enables the recording of HR-QoL.

3. source of information (self or external assessment)

The questionnaire tool tends to allow both forms of assessment. However, it is not yet clear whether the questionnaire was completed by the patient or a representative.

4. behavioral reference

The concept of using questionnaires restricts this axis of QoL survey the most. In order to integrate the behavioral reference of a patient, it is necessary to observe the patient over a longer period of time and detect dependent deviations for the individual. Questionnaires are originally designed to determine the tendency of cohorts rather than to detect the variation of an individual. To integrate this axis more closely, the concept would have to be adapted.

Up to now, compliance for questionnaires in medicine has only been measured within a certain period of studies. However, studies have shown that having a longitudinal survey of a health status through questionnaires leads to a decreasing compliance and an 
increasing response bias, such as a social desirability response bias which often occurs in self-report research. [2]

A combination of measuring and interpreting QoL though questionnaires and behavioral references would be desirable. After the usability study was well received, the next step will be to survey HR-QoL outside the clinical environment. Here, interoperability becomes even more important, as not only patient devices are integrated, but also the treatment network is expanded. A technical advancement to patientgenerated data on wearables in the everyday life of the patient poses a maximum challenge to the system in terms of flexibility and data security.

\section{Conclusion}

Questionnaires are a concept that has been used successfully in clinical studies for many years. However, with the change to personalized therapy and the longitudinal determination of QoL, it is necessary to develop new concepts. The behavioral reference plays an important role in measuring QoL in oncology as the answers of questionnaires are consciously or unconsciously distorted or influenced by the patient. It's also important to have a continuous measurement of wellbeing. In average, 3-4 symptoms are forgotten by the patient until the next consultation. Of these, about 2 symptoms have been reported as severe in the direct recall. [14]

In order to continuously integrate the behavioral reference, not only questionnaires can be used, but the patient must be constantly monitored and the behavior must be interpreted. With new technologies, a certain behavior, such as the physical activity of a person, can be measured by means of a pedometer and, with the help of machine learning algorithms, be related to the behavior of the last days/weeks. When selecting sensors, it is important to ensure that they are suitable for everyday use, have open interfaces and interpretable data transmission. It could be taken advantage of the spread of smartphones and wearables with their extensive sensor systems. In the research of behavioral disorders these concepts are already successfully used to detect manic phases early and to intervene. [15] A modification of this concept in combination with motivation through gamification elements takes the longitudinal collection of QoL data to a new level. The authors are currently investigating the effects of such an extension. New data security, data protection, but also ethical questions have to be clarified before such a system can be applied. The aim is to extend the survey of HR-QoL through questionnaires, not to replace it.

\section{Acknowledgements}

The authors at MOLIT Institute gGmbH thank the Dieter Schwarz Stiftung gGmbH for their support.

\section{Conflict of Interest}

The MOLIT Institute is a non-profit organization, funded by donation. The first author is a master student at the university of applied science in Heilbronn and employed part- 
time at the MOLIT Institute. The topic was further developed from the preliminary work of a bachelor thesis.

The last two authors are the founders of the MOLIT Institute.

\section{References}

[1] BULlingER, M.; PETERSEN, C.; MEHNERT, A. Erfassung der Lebensqualität in der Onkologie. In: Praxis der Viszeralchirurgie. Springer, Berlin, Heidelberg, 2006. S. 323-332.

[2] VAN DE MORTEL, Thea F., et al. Faking it: social desirability response bias in self-report research. Australian Journal of Advanced Nursing, The, 2008, 25. Jg., Nr. 4, S. 40.

[3] "WHOQOL: Measuring Quality of Life". World Health Organisation [Online] www.who.int/healthinfo/survey/whoqol-qualityoflife/en/ [accessed: 18.03.2020]

[4] BOTTOMLEY, Andrew, et al. Analysing data from patient-reported outcome and quality of life endpoints for cancer clinical trials: a start in setting international standards. The Lancet Oncology, 2016, 17. Jg., Nr. 11, S. e510-e514.

[5] BASCH, Ethan, et al. Symptom monitoring with patient-reported outcomes during routine cancer treatment: a randomized controlled trial. Journal of Clinical Oncology, 2016, 34. Jg., Nr. 6, S. 557.

[6] COOLBRANDT, A. et al. Use of a symptom diary during chemotherapy: A mixed-methods evaluation of the patient perspective, European Journal of Oncology Nursing, vol. 31, pp. 37-45, 2017.

[7] KANG, E. et al. The impacts of prognostic awareness on mood and quality of life among patients with advanced cancer, American Journal of Hospice and Palliative Medicine ${ }^{\circledR}$, p. 1049909120905789, 2020.

[8] BLACHETTA, Frederik, et al. Weiterentwicklung der eHealth-Strategie: Studie im Auftrag des Bundesministeriums für Gesundheit. 2016.

[9] EDIDIN, H. et al. HL7 for BizTalk. Apress, 2014.

[10] BRAUNSTEIN, M. L. et al. A brief history and overview of health informatics, in Health Informatics on FHIR: How HL7's New API is Transforming Healthcare, pp. 3-12, Springer, 2018.

[11] KBV, KBVWebsite, https://fhir.kbv.de/ [cited 08.07.20]

[12] MOLIT Institut gGmbH, MOLIT Github Repository, https://github.com/molit-institute/equ-pwa [cited 08.07.2020].

[13] MOLIT Institut gGmbH, MOLIT Github Repository, https://github.com/molit-institute/equ-manager [cited 08.07.2020].

[14] BREEN, Sibilah, et al. Remote real-time monitoring for chemotherapy side-effects in patients with blood cancers. Collegian, 2017, 24. Jg., Nr. 6, S. 541-549.

[15] PRIOR, Margot, et al. Longitudinal predictors of behavioural adjustment in pre-adolescent children. Australian and New Zealand Journal of Psychiatry, 2001, 35. Jg., Nr. 3, S. 297-307. 